\title{
Quality and reliability of information available on YouTube videos pertaining to transforaminal lumbar epidural steroid injections
}

\author{
Aysel Gürcan Atci ${ }^{1}$, Ibrahim Burak Atci ${ }^{2}$ \\ 1 MD. Specialist. Baltalimanı Bone and Joint Research and Education \\ Hospital, Department of Physical Therapy Rehabilitation, TURKEY \\ 2 MD. Assoc. Prof. Istanbul Training and Research Hospital, \\ Department of Neurosurgery, TURKEY
}

\begin{abstract}
Background. This study analyses the transforaminal lumbar steroid injection videos that have the highest views and likes on YouTube, and attempts to reveal the video qualities in order to contribute to the literature.

Methods. For review, "transforaminal lumbar steroid injection" was written to the standard YouTube search bar, and the videos with the highest views were ranked using advanced search preferences. The 50 most widely viewed videos were watched and scored by 2 physicians.

Results. The mean Modified DISCERN Score of the videos was 2,66+/-1,032 (the lowest: 1; the highest: 4) while the mean GQS score was 2,876+/-1,06 (the lowest: 1; the highest:4). In addition, the mean DISCERN score and the mean GQS value were 3,51 and 3,82, respectively, for the informational videos that were uploaded by health professionals but did not contain actual surgery.

Conclusion. We think that medical associations and state authorities in medicine should check the validity and accuracy of the information on the internet and should support the society in access to the most correct information.
\end{abstract}

\section{INTRODUCTION}

In daily practice, internet search rates have increased in almost every subject due to developing information technologies that are used more and more every day. In health practices, professionals and patients make internet researches in order to get information and gain experience. Among these sources of application, YouTube is the biggest video archive website in the world and attracts $95 \%$ of internet users with 30 million active users every day (1). There are also many healthrelated videos in the archive. Generally, patients apply to a physician and get detailed information about recommended treatments but they are also inclined to watch on YouTube the operation to be carried out.

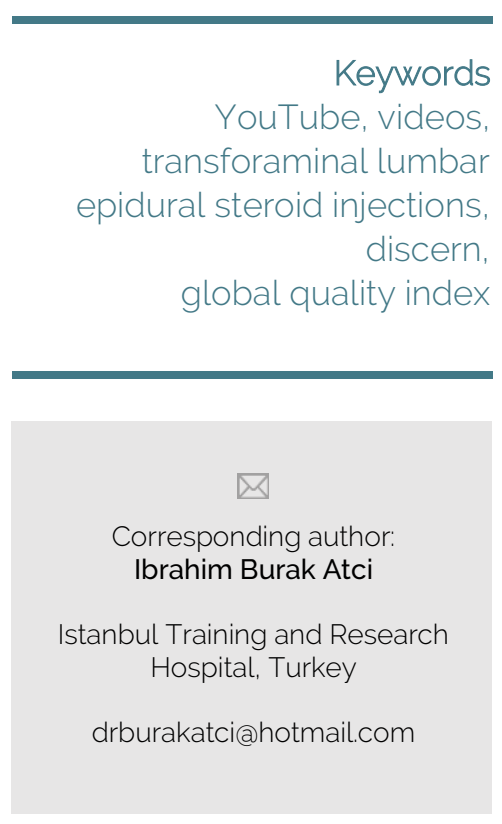

Copyright and usage. This is an Open Access article, distributed under the terms of the Creative Commons Attribution Non-Commercial No Derivatives License (https://creativecommons org/licenses/by-nc-nd/4.0/) which permits noncommercial re-use, distribution, and reproduction in any medium, provided the original work is

unaltered and is properly cited. The written permission of the Romanian Society of re-use or in order to create a derivative work.

ISSN online 2344-4959 (C) Romanian Society of Neurosurgery

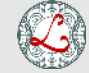

First published June 2019 by London Academic Publishing www.lapub.co.uk 
Therefore; the quality of a video, the persons who shot it and whether such video contains correct information are matters of great importance.

Lumbar disc herniation is frequently observed among people. Recently, fluoroscopy injections have been increasingly applied with success for nerve roots that cause pressure on patients whose pains do not regress with medical treatment and rehabilitation programs and who are not appropriate for operation (11).

This study analyses the transforaminal lumbar steroid injection videos that have the highest views and likes on YouTube, and attempts to reveal the video qualities in order to contribute to the literature.

\section{MATERIAL AND METHODS}

Search Strategy and Data Collection

For review, "transforaminal lumbar steroid injection" was written to the standard YouTube search bar, and the videos with the highest views were ranked using advanced search preferences. The 50 most widely viewed videos were watched and scored by 2 physicians.

\section{Inclusion and Exclusion Criteria}

The videos that were not in English language or did not have subtitles or speech or that did not explain the operation were eliminated.

\section{Variables Extracted}

Views, upload dates, like rates, uploaders, video lengths, comment numbers, like numbers and dislike numbers were identified as well as whether they were actual or animated videos.

In addition, video power index (VPI) values [(number of likes / number of likes $p$ number of dislikes) 100] were calculated to evaluate the popularity of the videos.

\section{Assessment of Usefulness}

All videos were independently evaluated by two physicians for usefulness and categorized into the following mutual exclusive categories.

1. Useful information: Videos designated as useful information were mainly focused on information delivery. They contained accurate information and were useful for learning how to do transforaminal lumbar steroid injection.

2. Misleading information: The videos contained in correctin formation or did not contain useful information about transforaminal lumbar steroid injection.

3. Useful patient opinion: The videos in this group have the DISCERN and GQS scores as 3 or above and clearly explain the patient experiences, the performance of operations, and preoperational and post operational pain scores.

4. Misleading patient opinion: The videos in this group have the DISCERN and GQS scores as 2 or lower and do not clearly explain patient experiences.

\section{Scoring System}

Video reliability was scored using a modified fivepoint DISCERN tool (2), which was adapted from the original DISCERN tool for the assessment of written health information by Charnock et al. (3).

The overall quality of each video was rate during the five-point Global QualityScale (GQS). The GQS was developed as an evaluation tool for website resources and it assesses the flow and ease of use of the information presented online, and the quality of video (Table 1) [4].

\section{Statistical analysis}

The results were statistically analysed during a nonparametric Kruskal-Wallis test. A p value of 0.05 or less was considered significant. The Statistical Package forth eSocial.

Sciencesversion 23 software (SPSS, Chicago, IL, USA) was used for all statistical analyses.

\section{RESULTS}

60 videos with the highest views were analyzed while 10 videos were later excluded from the analysis for they were neither in English language nor contained subtitles. Among these, 10 videos were animations while 32 consisted of actual images. There were 22 surgery, 10 patient view and 13 technically-narrated animated and actual videos. On the other hand, 6 videos were uploaded by physicians and gave theoretical information about the processes they applied (Figure 2). The oldest video was uploaded in 2007 while the newest one was added to the system in 2017. The videos were uploaded by hospitals ( 27 videos), health professionals and physicians (13 videos), and personal accounts (10 videos) (Figure 1).

The mean time of the video lengths was $4.22 \mathrm{sec}$ (the shortest: 0,20 sec; the longest: $12,08 \mathrm{sec}$ ), and the mean view was $88,293+/-9,75$ (the least viewed: 4075; the most viewed: 825.731 ). The daily mean 
view of the videos was $42,975+/-4,442$ (the least viewed: 4,45; the most viewed: 323,8 ). The mean like rate was 53,6+/-3,52 (the most liked:3000; the least liked: 0 ), and the mean dislike rate was $28+/-2,21$ (the most disliked: 758; the least disliked: 0).As for the comments, the mean number was 42,8 (the least commented: 0; the most commented: 423).Similarly, video power index (VPI) analyses showed that the mean VPI value of the 50 videos was 82,557+/-9,766 (the lowest VPI: 60,1; the highest VPI: 95,8).

The mean Modified DISCERN Score of the videos was 2,66+/-1,032 (the lowest: 1 ; the highest: 4 ) while the mean GQS score was 2,876+/-1,06 (the lowest: 1; the highest:4). In addition, the mean DISCERN score and the mean GQS value were 3,51 and 3,82, respectively, for the informational videos that were uploaded by health professionals but did not contain actual surgery. Similarly, the mean DISCERN score and the mean GQS value were 1,08 and 1,29, respectively, for the patient videos in which personal experiences were shared. No statistically significant correlation was found between the GQS and DISCERN scores according to both researchers and VPI values $(P>0.05)$.

TABLE 1. Analyses of video characteristics by usefulness category

\begin{tabular}{|l|l|l|l|l|}
\hline & $\begin{array}{l}\text { Useful Information } \\
\text { (Gr1) }\end{array}$ & $\begin{array}{l}\text { Misleading } \\
\text { Information (Gr2) }\end{array}$ & $\begin{array}{l}\text { Useful Patient } \\
\text { Opinion (Gr3) }\end{array}$ & $\begin{array}{l}\text { Misleading Patient } \\
\text { Opinion (Gr4) }\end{array}$ \\
\hline Video Number & $\mathrm{n}: 16(32 \%)$ & $\mathrm{n}: 24(48 \%)$ & $\mathrm{n}: 4(8 \%)$ & $\mathrm{n}: 6(12 \%)$ \\
\hline Views peer day & $35,2+/-7,31$ & $53,7+/-8,1$ & $40,3+/-4,55$ & $42,7+/-8,3$ \\
\hline Video Lenght & $\begin{array}{l}4,01 \min (2,04-5,91 \\
\mathrm{min})\end{array}$ & $\begin{array}{l}3,50 \min (0,20-8,17 \\
\mathrm{min})\end{array}$ & $\begin{array}{l}6,03 \mathrm{~min}(5,02-8,54 \\
\min )\end{array}$ & $\begin{array}{l}7,25 \mathrm{~min}(4,01-12,08 \\
\mathrm{min})\end{array}$ \\
\hline Like & $216+/-24$ & $27,14+/-2,1$ & $43,08+/-2,01$ & $33,4+/-3,33$ \\
\hline Dislike & $189,5+/-24,5$ & $46,3+/-4,44$ & $20,2+/-1,35$ & $15,2+/-1,56$ \\
\hline Comments & $26,8+/-2,33$ & $27,2+/-1,33$ & $92,1+/-7,41$ & $113,4+/-11,2$ \\
\hline Discern Score & 3,4 & 2,2 & 3,2 & 0,5 \\
\hline GQS Score & 4,1 & 2,3 & 4,2 & 1,6 \\
\hline
\end{tabular}

TABLE 2. Pairwise comparisons of video groups according to usefulness

\begin{tabular}{|l|l|l|l|l|l|l|}
\hline & Gr1-Gr2 & Gr1-3 & Gr1-4 & Gr2-3 & Gr2-4 & Gr3-4 \\
\hline $\begin{array}{l}\text { Discern } \\
\text { Score } p \\
\text { value }\end{array}$ & 0,518 & 0,708 & 0,001 & 0,652 & 0,332 & 0,0018 \\
\hline $\begin{array}{l}\text { GQS Score } \\
\text { p value }\end{array}$ & 0,125 & 1,00 & 0,001 & 0,069 & 0,852 & 0,001 \\
\hline
\end{tabular}

Values of $\mathrm{p} 0,05$ was accepted

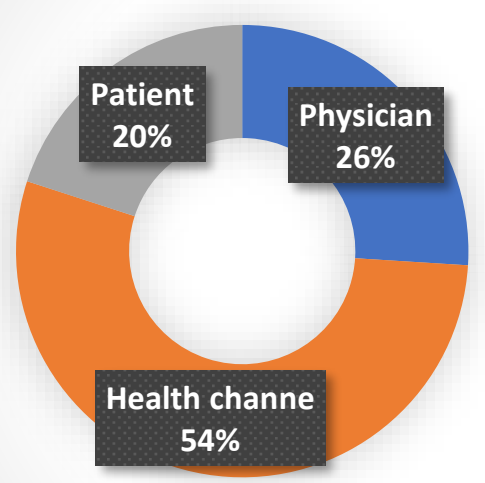

Physician

Health channe

Patient 


\section{Modify Discern (1 point per question answered yes)}

1. Is the video clear, concise, and understandable?

2. 2. Are valid sources cited? (from valid studies, physiatrists or rheumatologists)

3. 3. Is the information provided balanced and unbiased?

4. 4. Are additional sources of information listed for patient reference?

5. 5. Does the video address areas of controversy/uncertainty?

\section{Global quality scale}

1. Poor quality, poor flow, most information missing, not helpful for patients

2. Generally poor, some information given but of limited use to patients

3. Moderate quality, some important information is adequately discussed

4. Good quality good flow, most relevant information is covered, useful for patients

5. Excellent quality and excellent flow, very useful for patients

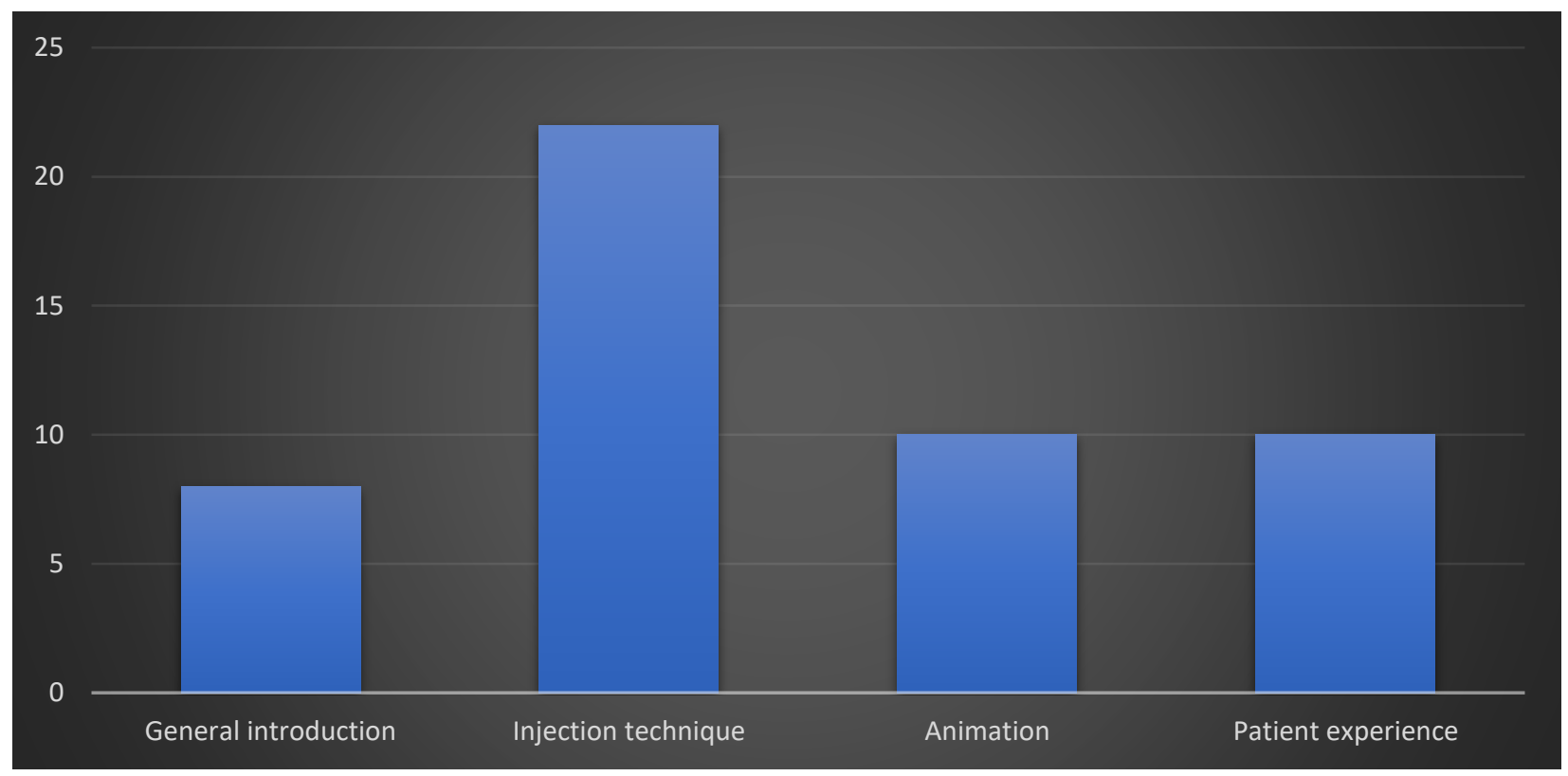

FIGURE 2. Video contents

\section{DISCUSSION}

YouTube is a video sharing website based in San Bruno, California, the USA. The website was founded in 2005 and was later acquired by Google in 2006. The primary purpose of the website is that the users can upload and share any video in any subject freeof-charge (5).

The first video uploaded to the website was the video showing Jawed Karim, one of the founders of the website, in which he appears in a zoo (5). Concerning medical subjects; health professionals, hospitals and patients have a heavier traffic of video uploading activity. Patients and patient relatives refer to these videos in order to see treatment methods and possible risks and to make inferences from the experiences of cases. On the other hand, health professionals use operation videos to learn by watching (10). However, there may also be incorrect, low-quality and prejudiced videos on this platform where everyone can upload videos free-of-charge without being subject to any inspection. PubMed reviews reveal 1089 studies that measure the quality of YouTube videos on health issues $(8,9)$. The first of these is a study from 2007 that evaluate the training of health professionals (6).

The first publication concerning transforaminal steroid application with fluoroscopy was made by Lutz GE in 1998. The study follows up 69 cases for about 80 weeks and describes the results thereto. The study was described as an effective attempt on cases with radiculopathy due to non-surgical disc herniation (7). After the article, PubMed also 
published a paper indicating that the attempt was a positive one. Many physicians who deal with pain studied this attempt and applied the same to their patients.

In this sense, the purpose of the present study is to find out whether patients can get reliable information from YouTube channels prior to their transforaminal operations applied to lumbar disc herniation cases that do not require or are not recommended surgery. The second purpose is to evaluate the quality of the theoretical and visual information health professionals can get from such videos. The literature review we made did not produce any study concerning the subject in question. There are various scales and measures to evaluate the quality of the information in videos and on the internet. In this study, 2 researchers assess the videos using modified DISCERN scoring system, Global Quality Index and Video Power Index (VPI). According to the analysis of the 50 videos with the highest views and VPIs, it was found out that the videos presented weak and poor-quality information to patients, patient relatives and professionals who desire to learn the narrated operation.

However, it was also observed that $80 \%$ of the videos were uploaded by health professionals and institutions. In 16 (32\%) videos with actual surgeries, it was seen that the average time was 3,44 seconds, the operators did not satisfactorily explain the methods before and after the operations, they did not clearly specify alternative treatments and effects and possible complications, and the videos were not supported with subtitles. It was observed that the videos did not explain the operations in simple language to convey the processes to patients and patient relatives but only the course was expressed, and that there were dialogs with patients during operations. Furthermore, it was revealed that 6 (12\%) videos with the highest results of evaluation were animated or notional surgery videos, made theoretical PowerPoint presentations and were supported with anatomic cross-sections.

Although 6 of 10 videos with patient experiences contained information about pain statuses in postop early stage and the post-op 2nd day, these were not found sufficient in terms of quality. The mostviewed 5 videos had approximately 300 views every day, on average, and 3 of these were actual surgeries while the other 2 were about patient experiences. The videos with the highest VPI values but had 2 or below in DISCERN and GQS scoring were found to convey inadequate information. In contrast with the foregoing, the videos with the highest scores had 60 views every day, on average, and did not appear on the first page when searched on YouTube. However, the videos that had the highest views but contained insufficient information appeared on top in YouTube searches. Apart from these, the video comment analyses demonstrated thatthe highest number of comments were entered to the uploads with patient experiences. The comments notably asked the regression rate of complaints, the length of the period without complaints and whether the operations were painful. Accordingly, the videos with the highest like numbers were those that contained patient remarks.

The videos were divided into 4 groups in terms of usefulness, and only 16 videos were found to contain useful and valid information. All these were uploaded by health professionals and were generally about physician remarks. The mean time of these videos was 2,44 seconds. Useful patient remarks were identified only in 4 videos, and their mean view time was 2,31 seconds.

The limitations of this study include the crosssectional design (popularity based on number of views changes constantly), and the inclusion of only the 50 most widely viewed videos (an arbitrary cut point).

\section{CONCLUSION}

As a result, it may not always be accurate to believe that the medical videos with high view, comment and like numbers on YouTube contain reliable, comprehensible and correct information. Although the access to information and videos on medical subjects is very easy in today's world, it is more appropriate to apply to experienced health professionals in order to get information. We think that medical associations and state authorities in medicine should check the validity and accuracy of the information on the internet and should support the society in access to the most correct information.

COMPLIANCE WITH ETHICAL STANDARDS

This study does not include any human participants or animals. Videos that were available to everyone were evaluated for this study. Therefore, ethics committee approval was not required. 


\section{REFERENCES}

1. YouTube. YouTube statistic page. https://www. omnicoreagency.com/youtube-statistics/.

Accessed October 25, 2018.

2. Singh AG, Singh $S$, Singh PP (2012) YouTube for information on rheumatoid arthritis-a wakeup call? J Rheumatol 39(5):899-903. https://doi.org/10.3899/jrheu m.111114 (Epub 2012 Apr 1).

3. Charnock D, Shepperd S, Needham G, Gann R (1999) DISCERN: an instrument for judging the quality of written consumer health information on treatment choices. J Epidemiol Community Health 53(2):105-111.

4. Bernard A, Langille M, Hughes S, Rose C, Leddin D, Veldhuyzen van Zanten S (2007) A systematic review of patient inflammatory bowel disease information resources on the World Wide Web. Am J Gastroenterol 102(9):2070-2077.

5. https:// tr . wikipedia.org/wiki/youtube history.
6. Skiba DJ (2007) Nurs Educ Perspect. Nursing education 2.0: YouTube 2007 Mar-Apr;28(2):100-2.

7. Lutz GE, Vad VB, Wisneski RJ .(1998) Arch Phys Med Rehabil. Fluoroscopic transforaminal lumbar epidural steroids: an outcome study. Nov;79(11):1362-6.

8. Ovenden CD, Brooks FM. Anterior cervical discectomy and fusion YouTube videos as a source of patient education. Asian Spine J. 2018;12:987-991.

9. Drozd B, Couvillon E, Suarez A. Medical YouTube videos and methods of evaluation: literature review. JMIR Med Educ. 2018;4:e3.

10. Tackett S, Slinn K, Marshall T, Gaglani S, Waldman V, Desai R. Medical education videos for the world: an analysis of viewing patterns for a YouTube channel. Acad Med. 2018;93:1150-1156.

11. Hassan KZ, Sherman Al.(2018)Epidural Steroids. StatPearls [Internet]. Treasure Island (FL): StatPearls Publishing; 2019 Jan-. 2018 Dec 19. 